\title{
A community of chemists and biologists
}

W e are pleased to present the inaugural issue of Nature Chemical Biology, a new international forum for the publication of high-impact research at the interface of biology and chemistry. Consistent with the diverse perspectives of its practitioners, 'chemical biology' has many definitions. In our view, chemical biology focuses on understanding biological systems at the molecular level and using these mechanistic insights to expand chemistry and biology in new directions. Chemical biology has emerged as a field grounded in technological advances brought about by the close collaboration of chemists and biologists. Using diverse experimental and theoretical techniques, chemical biologists have tackled challenging problems in biology, ranging from cellular signaling to drug development and neurobiology. Despite the expansive research interests of chemical biologists, the field is connected by a common desire to understand and manipulate living systems at the molecular level with increasing precision.

Since 1992, Nature Publishing Group has launched nine Naturetitled monthly journals, each of which has become a leading publication in its discipline. During this period, chemical biology has emerged as a research frontier on an international scale. Numerous universities have developed chemical biology programs that cross traditional departmental lines. Funding agencies worldwide have identified the chemistry-biology interface as a target for investment. For example, the NIH Roadmap (http://nihroadmap. nih.gov) underscores the importance of collaborations between chemists and biologists to the future of biomedical research. The increased number of chemical biology conferences highlights the importance of this interdisciplinary field for both industrial and academic scientists. Finally, from its roots in bioorganic chemistry, the field has grown to include biologists and chemists from across traditional disciplines. For example, biologists, equipped with quantitative chemical tools, have expanded our understanding of molecular interactions within cells. Physical, inorganic and analytical chemists, who have changed the way we perceive biology, find themselves in the mainstream of chemical biology research.

Given these exciting developments, we believe that chemical biology deserves greater visibility in the scientific community. Our primary aim in launching Nature Chemical Biology is to create a top-tier international journal that reflects the diversity and excitement of chemical biology research. We will strive to reach out to the chemistry and biology communities equally and deliver monthly issues that will spur conversations between these traditional disci- plines. To achieve this, we will publish research papers from across chemical biology, chosen for their exceptional quality and scientific impact. Each monthly issue will also include enhanced content such as reviews, research highlights and News \& Views articles that put important developments in context for a broad readership.

The scope of Nature Chemical Biology includes four themes of contemporary chemical biology. Chemical Synthesis, including biosynthesis, remains an enabling technology in the field. From its roots in biochemistry, Chemical Mechanisms in Biology focuses on understanding natural biological systems using chemical and biological tools. Expanding Biology through Chemistry applies chemical techniques to increase the range of biological systems, while Expanding Chemistry through Biology emphasizes how biological diversity and information may be harnessed in the service of chemistry. This first issue of the journal reflects its broad scope by including topics ranging from bacterial to human biology and from small molecules to ribozymes.

As a Nature publication, we share editorial criteria with Nature and the other research journals. We are committed to a fair and rapid peer review process in which we select papers of significance to a broad readership. Like the other Nature journals, Nature Chemical Biology does not have an external editorial board. Instead, manuscript decisions are made by a professional editorial team of chemical biologists in consultation with experts in the field. This approach has served Nature journals well over the years by allowing editorial flexibility and ensuring that viewpoints from across the field are heard. Despite these shared policies, Nature Chemical Biology is editorially independent from the other Nature titles. We consider each manuscript on its merits, focusing on its appropriateness for the scope of the journal, its potential significance within the field and its likely interest to a general audience of chemists and biologists. The editorial team places a high premium on manuscripts that report significant conceptual or methodological advances and that demonstrate the biological relevance of their results.

The editorial team has thoroughly enjoyed bringing this first issue of Nature Chemical Biology to you, our readers. We believe that Nature Chemical Biology will become the primary source for the highest-impact research and commentary in chemical biology by offering an exceptional forum for the ongoing conversations between chemists and biologists. We look forward to working with the chemical biology community to achieve this important goal. 